\title{
Edge Eigenface Weighted Hausdorff Distance for Face Recognition
}

\author{
Huachun Tan* \\ Department of Transportation Engineering, Beijing Institute of Technology, Beijing, 100081, China \\ Email: \{tanhc \}@bit.edu.cn \\ Yu-Jin Zhang \\ Department of Electronic Engineering, Tsinghua University, Beijing, 100084, China \\ Email: zhang-yj@mail.tsinghua.edu.cn \\ Wuhong Wang, Guangdong Feng, Hui Xiong \\ Department of Transportation Engineering, Beijing Institute of Technology, Beijing, 100081, China \\ Email: \{ wangwuhong, 20903201, xionghui \}@bit.edu.cn \\ Jie Zhang \\ Research Institute of Highway, Ministry of Transport of China, Beijing, 100088, China \\ Email: zj03410401@126.com \\ Yong Li \\ Department of Electrical Engineering, Notre Dame University, \\ Email:yli5@nd.edu \\ Received 20 July 2011 \\ Accepted 25 November 2011
}

\begin{abstract}
The different face regions have different degrees of importance for face recognition. In previous Hausdorff distance (HD) measures, points are treated as same importance, or weight different points that calculated from gray domain. In this paper, a new weighting function of HD based on the eigenface from edge domain, which reflects the discriminative properties of face edge images effectively, is proposed for face recognition. Experiments show the proposed method outperforms previous HD measures.
\end{abstract}

Keywords: Face Recognition; Hausdorff Distance; Weighting Function; Eigenface

\section{Introduction}

In the past decades, face recognition has received significant attention ${ }^{1-3}$. It has applied in wide range of commercial and law enforcement applications. In intelligent transportation system, face recognition systems are widely used for drivers' licenses and vehicular access ${ }^{1}$. Many methods have been proposed. However, various changes in face images, such as facial expression, occlusion, and illumination, make the process more difficult.

Psychological studies have indicated that line drawings of objects can be recognized as quickly and almost as accurately as photographs ${ }^{4}$. The evidence

\footnotetext{
*Corresponding author. Tel.: +86-10-6891-4582. Email: tanhc@bit.edu.cn (Huachun Tan). 
implicates that the process of face detection and face recognition may start at the level of early vision where edge-like retinal images of faces are screened at a very high speed, thus reducing the computation complexity needed to perform high-level cognitive function ${ }^{5}$. The advantage of using edges as image features is that they can provide robustness to illumination change and simplicity of presentation.

The Hausdorff distance is an efficient measure for binary image comparison. In recent years, many modified Hausdorff distance measures have been proposed to improve the performances in terms of efficiency or accuracy, and applied them to various applications $^{5-10,12-18}$.

In human face analysis, it is usually assumed that the different facial points or facial regions have different degrees of importance ${ }^{5}$. For example, the eyes, mouth, face contour, etc. have higher degree of importance than other face regions, because the edge points of the eyes, mouth and face contour can reflect the structure of human face more effectively. Most of previous Hausdorff distance measures, such as Classic Hausdorff distance (CHD) ${ }^{6}$, Modified Hausdorff distance (MHD) ${ }^{7}$ and "Doubly Modified Hausdorff distance" (M2HD) ${ }^{8}$ do not consider the relative importance among different facial regions, nor make distinction between the different parts of the face.

Spatially Weighted Hausdorff distance (SWHD) ${ }^{9}$ and Spatially Eigen-Weighted Hausdorff distance (SEWHD) $^{5}$ assign different weighting factors for different facial regions in computing the distance for face recognition, in which the weighting functions are defined according to the representation of gray domain. Hausdorff distance is a measure for two binary point sets, not for grayscale point sets. A point which is important in grayscale domain does not necessarily mean it is important in edge domain. Consequently, it is more suitable to define the weighting function according to the properties of face edge images.

In our previous work, Weighted Hausdorff distance $^{10}$ (we call it EFWHD in the following.), in which the weighting function is computed from the edge points appearing frequency, is proposed for face localization. However, for face recognition, the weighting function should be able to reflect the discriminative properties among different face edge images.
In this paper, a new weighted Hausdorff distance measure is proposed for face recognition. In the new measure, the weighting function is generated based on the eigenface of edge images, not the eigenface of grayscale images as proposed in ref. 5, nor the edge points appearing frequency in ref.9. Therefore, the weighting function can reflect the discriminative properties of face edge images effectively. Based on the proposed weighting function, a new Hausdorff distance measure termed Edge Eigenface Weighted Hausdorff distance (EEWHD) is applied to face recognition. The performances of proposed Hausdorff distance measure was evaluated on Yale face database ${ }^{11}$, and were compared with previous ones. Experimental results show that the proposed weighted Hausdorff distance measure achieves higher recognition rate than previous ones.

The rest of the paper is organized as follows. In Section 2, some Hausdorff distance measures are briefly overviewed. The proposed weighted Hausdorff distance and its application to face recognition are described in Section 3. In Section 4, the experimental results are illustrated to show the superiority of proposed measure. And conclusions are given in Section 5.

\section{Overview of Hausdorff Distance}

\subsection{Classic Hausdorff Distance}

Given two finite point sets $A=\left\{a_{1}, \ldots, a_{p}\right\}$ and $B=\left\{b_{1}, \ldots, b_{q}\right\}$, the classic Hausdorff distance (CHD) is defined as

$$
H(A, B)=\max (h(A, B), h(B, A))
$$

where

$$
h(A, B)=\max _{a \in A} \min _{b \in B}\|a-b\|
$$

and $\|a-b\|$ is the Euclidean distance between $a$ and $b$.

The function $h(A, B)$ is called the directed Hausdorff distance from $A$ to $B$. It identifies the point $a \in A$ that is the farthest from any point of $B$ and measures the distance from $a$ to its nearest neighbor in $B$. The Hausdorff distance $H(A, B)$ is the maximum of $h(A, B)$ and $h(B, A)$. Consequently, it is able to measure the degree of mismatch between two sets by measuring the distance of the point of $A$ that is farthest from any point of $B$, and vice versa. 


\subsection{Some modified Hausdorff distances with no weighting function}

A number of modified Hausdorff distance measures ${ }^{6-17}$ have been proposed which provide a more reliable and robust measure than the original one. Dubuisson ${ }^{8}$ proposed a modified Hausdorff distance (MHD) measure for object matching. MHD was also applied to face localization by Josorskey et $\mathrm{al}^{12}$. The directed MHD is defined as

$$
h(A, B)=\frac{1}{N_{a}} \sum_{a \in A} \min _{b \in B}\|a-b\|
$$

where $N_{a}$ is the number of points in $A$.

Takacs $^{9}$ introduced the notion of neighborhood function and associated penalty to 'doubly' modified Hausdorff distance (M2HD). This directed M2HD is defined as

$$
h(A, B)=\frac{1}{N_{a}} \sum_{a \in A} d(a, B)
$$

where

$$
d(a, B)=\max \left(I * \min _{b \in N_{B}^{q}}\|a-b\|,(1-I) P\right)
$$

In this formulation, $N_{B}^{a}$ is the neighborhood of point $a$ in set $B$. $I$ is an indicator, which is equal to 1 if there exists a point $b \in N_{B}^{a}$, and 0 if otherwise.

Many other measures were proposed to alleviate the sensitivity of the Hausdorff distance to outlier points and occlusion, or can fast the matching process. Huttenlocher et $\mathrm{al}^{7}$ proposed a partial Hausdorff distance (PHD) measure for comparing partial portions of images containing severe occlusions or degradations. Sim et $\mathrm{al}^{13}$ proposed two robust HD measures based on M-estimation and least trimmed square for object matching which are robust to outliers and occlusions. Zhu et $\mathrm{al}^{14}$ proposed weighted modified Hausdorff distance (WMHD) in the circular Gabor feature space to determine which position can be possible object model location. Gao et $\mathrm{al}^{15}$ introduced a new conception of Hausdorff distance based on line segmentation for face recognition and achieved higher recognition rate. Gao ${ }^{16}$ proposed a modified Hausdorff distance based on dominant point to fast the matching process and reduce the memory requirement. $\operatorname{Han}^{17}$ proposed Hausdorff distance with explicit paring (HDEP) by considering the candidate of correspondence for 3D object recognition. Zhao $^{18}$ proposed a new Hausdorff distance by transforming the binary image to gray image to reduce the sensitivity of noisy and outliers. Chen ${ }^{3}$ proposed a novel face image similarity measure based on Hausdorff distance, which is applied to feature space, not the points set. In these measures, they do not consider the relative importance between the different face regions.

\subsection{Weighted Hausdorff distance for face analysis}

In the application of face analysis, some HD measurements are proposed by considering the relative importance between the different face regions.

Lin et $\mathrm{al}^{6}$ proposed spatially weighted Hausdorff distance (SWHD) and spatially eigen-weighted Hausdorff distance (SEWHD) by considering the importance of different face regions. The directed Hausdorff distances of both SWHD and SW2HD have the same form

$$
h(A, B)=\frac{1}{N_{a}} \sum_{a \in A} w(b) \min _{b \in B}\|a-b\|
$$

where $w(b)$ is weighting function. For SWHD, the weighting function is determined manually according to the spatial information of face. For SEWHD, the weighting function is generated by the first eigenface of the training set. When the two weighting functions incorporate with M2HD, the directed Hausdorff distances are called spatially weighted double modified Hausdorff distance (SWM2HD) and spatially eigenweighted doubly modified Hausdorff distance (SEW2HD).

However, though the weighting functions of SWHD and SEWHD treat different face regions with different importance, they have some problems when applied to face localization as described in Section 1. A simple example to illustrate the problems is also shown in the Section 3.

$\operatorname{Tan}^{10}$ proposed EFWHD for face localization, in which the weighting function is calculated by the edge points appearing frequency. Thus, the weighting function can reflect the common properties of different face edge images, and is suitable for face detection. However, for face recognition, the aim is to distinguish faces of different persons. The weighting function should be able to reflect the discriminative properties among different face edge images. 


\section{Face recognition using new weighted Hausdorff distance}

\subsection{Edge Eigenface Weighted Hausdorff distance}

In face recognition, the weighting function of Hausdorff distance should be able to represent the discriminative properties of the edge images of human face. The sizes of all faces in the training set can be normalized firstly. Then, the proposed weighting function can be generated by the first eigen-vector of the edges of human face, that is

$$
w(x, y)=\left(\frac{255}{\max (v)-\min (v)}\right) v(x, y)
$$

where $v(x, y)=\left|v_{1}(x, y)\right|$, and $v_{1}(x, y)$ is the first eigenvector of the face edge images. $\max (v)$ and $\min (v)$ represent the maximum and minimum magnitudes of $v(x, y)$. We call the Hausdorff distance using the proposed weighting function as Edge Eigenface Weighted Hausdorff distance (EEWHD) in the following.

The definition of weighting function is very similar to that of $\mathrm{SEWHD}^{5}$ and EFWHD ${ }^{10}$. However, the proposed weighting function is based on the first eigenface of edge images, while the weighting function of SEWHD is based on the first eigenface of grayscale images, the weighting function of EFWHD is generated from the edge points appearing frequency which reflect the common properties of different faces.

We illustrate the idea of proposed weighting function, and compare it with the weighting function used in SEWHD and EFWHD by a simple example shown in Fig. 1, in which the original images are assumed to be simple structures of human face. Since different persons have different sub-structures in the face, we can suppose there are two classes of face, as shown in Fig. 1. Then, the first eigen-vector of grayscale images and that of edge images are calculated. We can see that the first eigen-vector of grayscale images can reflect the essential difference between the two classes in grayscale domain, but fail to reflect the essential difference between the two classes in edge domain. The edge appearing frequency can reflect the common properties of the edge images effectively, but fail to represent the discriminative properties between the two edge images. However, the proposed weighting function can reflect the essential difference between the two classes in edge domain effectively. Our goal is to distinguish the two classes in edge domain, neither to do in grayscale domain, nor to detect the two classes. In terms of face recognition using face edge images, we think using the eigen-vector of edge images as the weighting function is more suitable.

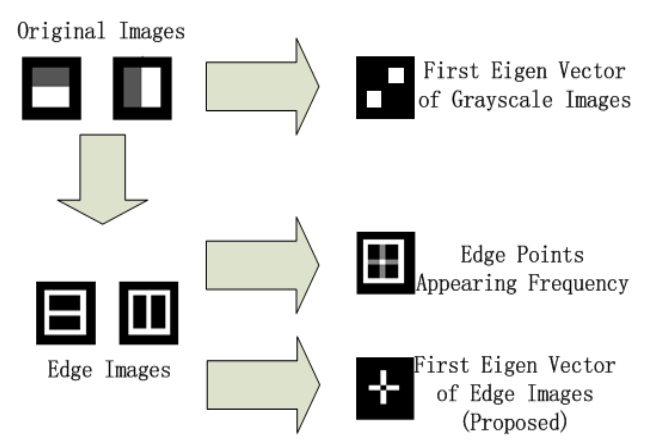

Fig. 1 A simple example of different weighting functions

Fig. 2 and Fig.3 give examples of false matching using the weighting function of SEWHD and EFWHD to distinguish the two classes. For simplicity, the edge images of the two classes are compared, one as model and the other as test image. In the process of matching using SEWHD, which is shown in Fig.2, the Hausdorff distance of the model and the edge of the test image is 0 , which means the model matches the test image. And the test image is determined as the wrong class. In Fig.3, it also can be found that the Hausdorff distance calculated by EFWHD is very small. Thus, the test image is apt to be classified to wrong class. The wrong classification of the two examples is due to the unsuitable weighting function. However, if using the proposed weighting function, the Hausdorff distance of the model and the edge of test image is not zero, nor small value, and therefore, can indicate that the test image is not as same class as that of the model if a suitable threshold is set.

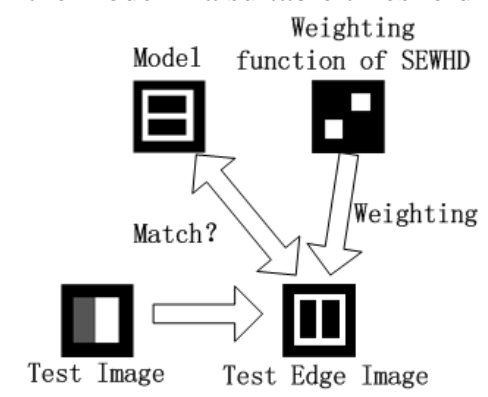

Fig. 2 Example of wrong matching using SEWHD 


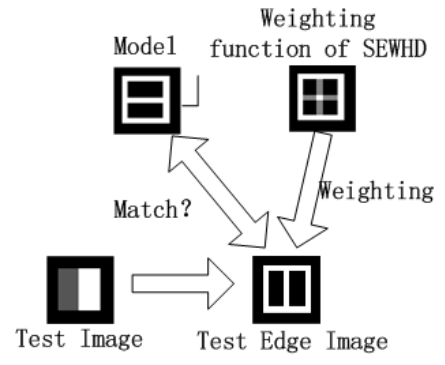

Fig. 3 Example of wrong matching using EFWHD

\subsection{Applied to face recognition}

The proposed Edge Eigenface Weighted Hausdorff distance (EEWHD) is applied to face recognition. Fig. 4 shows the framework of face recognition system. The preprocess is as same as that in ref. 8 and the others is as same as that in ref. 5. The following describes each process briefly.

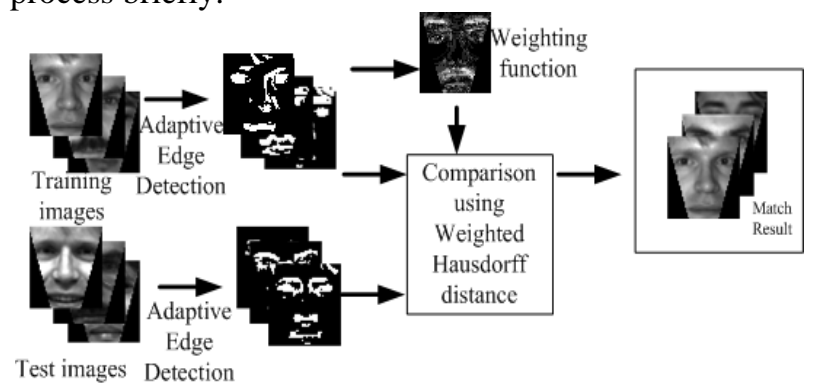

Fig. 4 Framework of face recognition system

In our experiments, Yale database ${ }^{11}$ is tested. Since all images in Yale database are normalized in scale, the normalization process is neglected.

To extract face edges, the process proposed in ref. 5 was used. The edge image $\mathrm{E}(\mathrm{x}, \mathrm{y})$ is firstly obtained by morphological operations. Then it is adaptively binarized. In the approach, the threshold to obtain the binary edge image is selected adaptively for each image so that the pixel ratio of ON vs. OFF bits in the image corresponded to a preset percentage ${ }^{7}$.

After the face edge images in training set are obtained, the eigen-vector of face edge images are computed. Then, the weighting function of proposed Hausdorff distance measure can be calculated according to Eq. (7). The eigen-vector used in system is also shown in Fig. 4.
Given a test face image, the face edge image can be obtained using the same process as that in training process. Then, the edge image of test face is compared to all face models in the training set using EEWHD. The results are ranked according to the value of EEWHD.

\section{Experimental Results and Discussions}

The proposed method was implemented with Matlab. We tested the performance of the proposed Hausdorff distance measure, and compared it with other Hausdorff distance measures for face recognition.

\subsection{Experimental setup}

The proposed method was tested on Yale database ${ }^{11}$. Yale database consists of 165 grayscale images of 15 different persons. There are 11 images per subject, one per different facial or configuration: enter-light, w/glasses, happy, left-light, w/no glasses, normal, rightlight, sad, sleepy, surprised, and wink.

This database features a larger variety of illumination, facial expression, and occlusion.

In the process of generating face model, one image of each person in the Yale database, totally 15 face images, are selected. The remaining face images, totally 150 face images, are used for testing.

\subsection{Experimental results}

To show the superiority of the proposed method, we compare the proposed distance (EEWHD) with other Hausdorff distance measures. Some Hausdorff distances mentioned above are investigated including,

- $\quad$ Classic Hausdorff distance (CHD)

- $\mathrm{MHD}^{7}$

- SEWHD $^{5}$

- EFWHD $^{10}$

All of these methods use the same pre-process as the proposed method, and the same binary face model was applied.

When calculating SEWHD, the weighting function generated from the first eigenface of grayscale images are obtained from the same training set as that for the proposed method. Also, the weighting function of EFWHD is obtained from the same training set as well.

In the first experiment, the images which are 'no glasses' are selected as training set. Others are used for testing. The pixel ratio is set to $19 \%$.The accumulative 
recognition rates of these measures are shown in Fig.5. From Fig. 5, it can be seen that EEWHD outperforms other measures, which achieves recognition rate of 91.3\%, 94.0\% and $96.7 \%$ for the first one, the first three and the first five likely matched faces, respectively. The reason is that the weighting function is based on the first eigen-vector of face edge images, and hence can discriminate the essence of edge images of different subjects. Although EFWHD is proposed for face localization, it works pretty well for face recognition. However, the recognition rate of EFWHD is 89.3\%, lower than that of the proposed method EEWHD 91.3\%. The underlying reason is that the weighting function for EFWHD is obtained in edge domain, but unlike EEWHD it does not incorporate or represent the discriminative information among different faces.

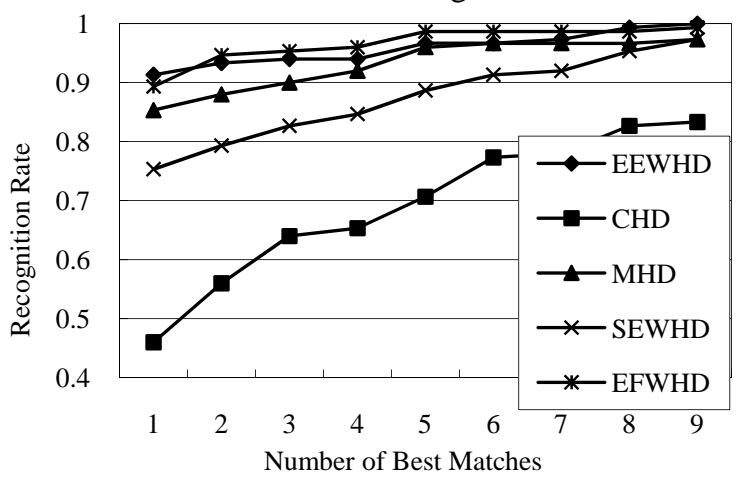

Fig. 5 Comparison of the accumulative recognition rates of different Hausdorff distance measures

SEWHD calculates PCA on grayscale images and the weighting function encodes the information about gray image structure. However, the recognition rate of SEWHD is $75.3 \%$ due to its inefficiency to represent the edge structure of images. In fact, the accumulative recognition rates of SEWHD are 75.3\%, 82.7\%, 88.7\% for the first one, three, five matched faces, which are evidently lower than that of EEWHD, and even lower than that of MHD.

In the second experiment, the images which are 'no glasses' in Yale database are selected as training set. Others are used for testing. The pixel ratio varies from 0.15 to 0.25 to test the influence of the pixel ratio on the performance of the proposed algorithm.

The performances of these methods are reported in Fig. 6. From Fig. 6, it can be observed that the recognition rates of EEWHD are higher than others in most tests. The best performance of EEWHD is achieved when pixel ratio is set to 0.19 , where the recognition rate is $91.3 \%$, while the best performances of CHD, MHD, SEWHD, and EFWHD are 52\%, 85.3\%, $76.0 \%$ and $90 \%$, respectively. Furthermore, the average recognition rate over these 11 tests (pixel ratio from 0.15 to 0.25 ) of CHD, MHD, SEWHD and EFWHD are $46 \%, 83.5 \%, 74 \%$ and $88.5 \%$ respectively, lower than that of EEWHD 88.9\%.

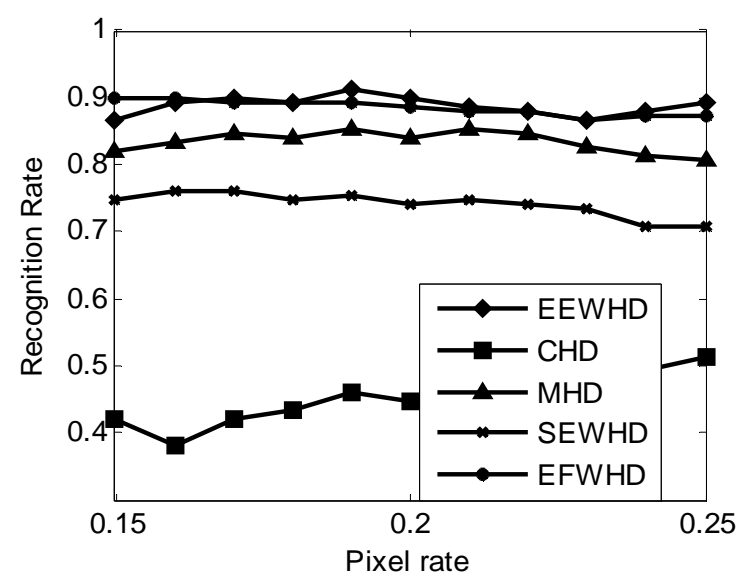

Fig. 6 Comparison of recognition rates using different pixel rate set

In the third experiment, one of 11 types of images is selected as training set, and other types or images are used as testing set. Since there are 11 types of images in Yale database, the test is performed 11 times, with each type being the training set for each time. Fig. 7 shows the experimental results where the pixel is set to 0.19 .

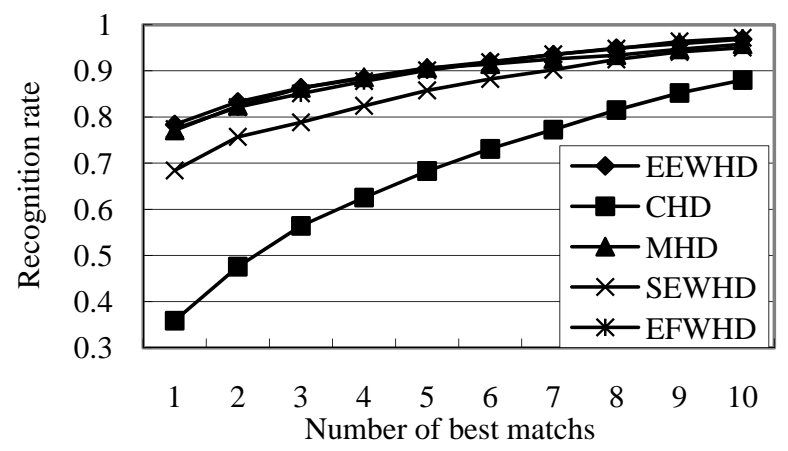

Fig. 7 Comparison of average accumulative recognition rate using different training set

EEWHD outperforms other Hausdorff distance measures in terms of the average recognition rate over the 11 tests. The average recognition rates for the first 
one, the first three and the first five likely matched faces for EEWHD are $78.4 \%, 86.3 \%$ and $90.6 \%$, respectively. While they are $77.4 \%, 85 \%$ and $90.1 \%$ for EFWHD, $77.1 \%, 86.3 \%$ and $90.6 \%$ for MHD, $68.4 \%, 78.9 \%$ and $85.8 \%$ for SEWHD, 35.9\%, 56.4\% and 68.3\% for CHD. This comparison shows that the proposed algorithm is more robust against differenct training sets than other methods when the pixel rate is 0.19 .

The fourth experiment extends the third experiment and the performances of these methods using different type of images as training data when the pixel ratio varies from 0.15 to 0.25 are tested. The statistic results are shown in Fig. 8. EEWHD also produces best performances in terms of average recognition rate over different training types of images. It can be seen that EEWHD outperforms other methods at every pixel ratio. Also, the average of the average recognition rate over different pixel ratio for EEWHD is $77.0 \%$, slightly higher than 76.9\% for EFWHD. However, the average recognition rate of EEWHD is much higher than that of MHD, SEWHD and CHD (75.7\%, 66.7\% and 38.1\%).

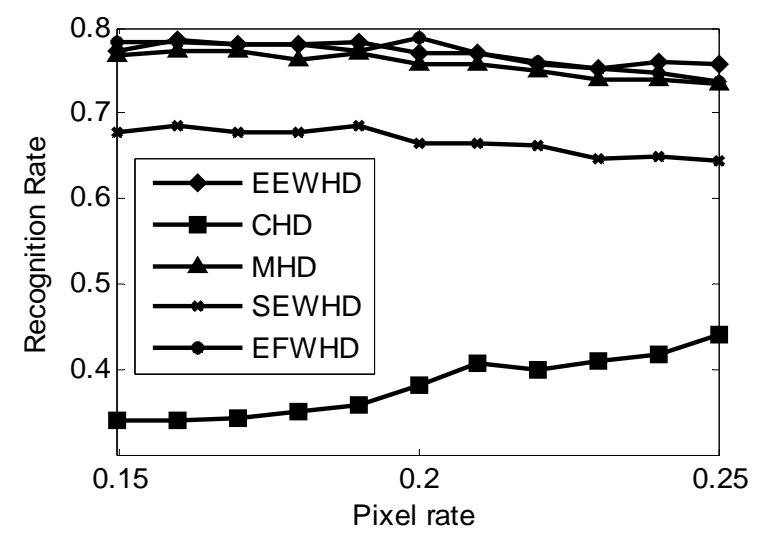

Fig. 8 Comparison of average recognition rates using different pixel ratio and different training set

Again, this experimental result confirms that both EEWHD and EFWHD perform significantly better than CHD, MHD, and SEWHD since both of them utilize the edge properties. The weighting functions of EEWHD and EFWHD are close to each other in Yale database and hence they give similar recognition rates.

Now we compare the highest average recognition rates (i.e., the maximum taken over all pixel rates) over different types of training images. EEWHD achieves the maximum $78.4 \%$ at the pixel ratio 0.19 , slightly lower than $78.8 \%$ by EFWHD achieving maximum at pixel ratio 0.20 . MHD, SEWHD, CHD achieve maxima at pixel rate $0.19,0.16,0.25$, respectively, and giving the highest recognition rate $77.1 \%, 68.4 \%, 44 \%$, which are lower than both EEWHD and EFWHD.

In all tests in spite of pixel ratio and the type of training set, EEWHD's best recognition rate is 91.3\% (pixel ratio $=0.19$ and using 'noglasses' for training). For MHD, SEWHD and EFWHD, the highest recognition rate are $88 \%, 76 \%$ and $90.7 \%$ respectively, all obtained when using 'normal' as training set and set pixel ratio as 0.19 . CHD achieves its best performance $54.7 \%$ when pixel ratio is 0.22 and using 'normal' for training.

As mentioned above, EEWHD achieves the best performances in the experiments. The performances of using EEWHD are slightly better than that of EFWHD, and much higher than those of SEWHD, MHD and CHD. The experimental results show that the proposed method can work well using different types of training set, including different types of illumination. This is because the weighting function of EEWHD can reflect the discriminative structures of different persons in edge domain.

\subsection{Discussions}

In our experiments, the performances obtained by using SEWHD ${ }^{3}$ are even lower than those of MHD. One of the reasons is the roughness of pre-process in our experiments. However, a more important reason is that the weighting function of SEWHD, which is based on the first eigenface of grayscale images, can not reflect the discriminative properties of edge images. A point which is important in grayscale domain does not necessarily mean it is also important in edge domain. From the experimental results, it is also found that the performances of $\mathrm{EFWHD}^{10}$ are slightly lower than EEWHD since its weighting function is generated in edge domain. Consequently, we think it is more suitable to define the weighting function according to the properties of face edge images.

\section{Conclusion}

In this paper, a new weighting function of Hausdorff distance for face recognition is proposed. The weighting function is generated based on the first eigenface of 
edge images, neither the first eigenface of grayscale images proposed in $\mathrm{SEWHD}^{3}$, nor the edge points appearing frequency in $\mathrm{EFWHD}^{10}$.

Since the proposed weighting function incorporates the discriminative properties of face edges more effectively, EEWHD outperforms other Hausdorff distance measures in face recognition applications. Experiments on Yale face database show the superiority of the proposed method over the previous ones. The best performance is achieved by EEWHD, where the recognition rate for the first one, the first three and the first five likely matched faces are 91.3\%, 94.0\% and $96.7 \%$, respectively. The highest average recognition rate over different types of training set for EEWHD is $78.4 \%$ at the pixel ratio 0.19 , which is slightly lower than EFWHD but significantly higher than MHD, SEWHD and CHD. Furthermore, EEWHD produces best average recognition rate using different types of images as training data and varying the pixel ratio from 0.15 to 0.25 . The average recognition rate of EEWHD is $77.0 \%$, outperforming EFWHD, MHD, SEWHD, and CHD.

Since both the weighting functions of EEWHD and EFWHD are obtained in edge domain, and very close to each other in Yale database, EEWHD and EFWHD give similar results. Unlike EEWHD, EFWHD does not incorporate or represent the discriminative information among different face edge images, and hence it performs slightly worse than the proposed method.

In our experiments, the recognition rate may be affected by pre-process. The recognition rate can further be improved by extracting face edges more effectively in the pre-process stage. This is our future work.

\section{Acknowledgements}

This work was supported by national natural science foundation of china (60872084 and 50878023), Fundamental research foundation of Beijing institute of technology (20090342015).

\section{References}

1. W. Zhao, R. Chellappa, P.J. Phillips and A. Rosenfeld, Face Recognition: A Literature Survey, ACM Computing Surveys, 35(4) (2003), 399-458.

2. N.K. Narayanan and V. Kabeer, Face Recognition Using Nonlinear Feature Parameter and Artificial Neural
Network, International Journal of Computer Intelligence Systems, 3(5) (2010), 566-574.

3. S. Chen, and B. C. Lovell, Feature space Hausdorff distance for face recognition. In 20th International Conference on Pattern Recognition, (2010), 1465-1468.

4. I. Biederman and J. Gu, Surface versus edge-based determinants of visual recognition, Cognitive Psychology, 20 (1998), 38-64.

5. K.H. Lin, K.M. Lam and W.C. Sui, Spatially eigenweighted Hausdorff distances for human face recognition, Pattern Recognition, 36 (2003), 1827-1834.

6. D.P. Huttenlocher, G.A. Klanderman and W.J. Ruchlidge, "Comparing images using the Hausdorff distance, IEEE Transaction on Pattern Analysis and Machine Intelligence, 15(9) (1993), 850-863.

7. M. Dubuisson and A.K. Jain, A modified Hausdorff distance for object matching, In Proc. 12th International Conference on Pattern Recognition, (1994), pp. 566-568.

8. B. Takacs, Comparing face images using the modified Hausdorff distance, Pattern Recognition, 31(12) (1998), 1873-1880.

9. K.H. Lin, B. Guo, K.M. Lam and W.C. Siu, Human face recognition using a spatially weighted modified Hausdorff distance, In Proc. the International Symposium on Intelligent Multimedia, Video and Speech Processing, (2001), pp.477-480.

10. H. Tan and Y. Zhang, A novel Hausdorff distance for face localization, Image and Vision Computing, 24 (2006), 656-662.

11. Yale University Face Database, http://cvc.yale.edu/projects/ yalefaces/yalefaces.html.

12. O. Josorsky, K.J. Kirchberg and R.W. Frischholz, Robust face detection using the Hausdorff distance, In Proc. Third International Conference on Audio- and Videobased Biometric Person Authentication, (2001), pp. 9095.

13. D.-G. Sim, O.-K. Kwon and R.-H. Park, Object matching algorithm using robust Hausdorff distance measures, IEEE Transaction on Image Process, 8 (1998), 425-429.

14. Z. Zhu, M. Tang and H. Lu, A new robust circular Gabor based object matching by using weighted Hausdorff distance, Pattern Recognition Letters, 25 (2004), 515-523.

15. Y. Gao and M.K.H. Leung, Line segment Hausdorff distance on face matching, Pattern Recognition, 35(2) (2002), 361-371.

16. Y. Gao, Efficiently comparing face images using a modified Hausdorff distance, IEE Proceedings Vision, Image and Signal Processing, 150 (2003), 346-350.

17. I. Han, Dong Yun and S.U. Lee, Modified Hausdorff distance for model-based 3-D object recognition from a single view, Journal of Visual Communication \& Image Representation, 15(1) (2004), 27-43.

18. C. Zhao, W. Shi and Y. Deng, A new Hausdorff distance for image matching, Pattern Recognition Letters, 26 (2005), 581-586. 